Highly Energetic Physical Processes and

Mechanisms for Emission from Astrophysical Plasmas

IAU Symposium, Vol. 195, 2000

P. C. H. Martens, S. Tsuruta, and M. A. Weber, eds.

\title{
Active Late-Type Stellar Coronae: Hints for Flare Heating?
}

\author{
M. Audard ${ }^{1}$ and M. Güdel ${ }^{1}$ \\ Paul Scherrer Institute, 5232 Villigen PSI, Switzerland
}

J. J. Drake and V. Kashyap

Harvard-Smithsonian Center for Astrophysics, Cambridge, MA 02138, U.S.A.

E. F. Guinan

Dept. of Astronomy \& Astrophysics, Villanova University, Villanova, PA 19085, U.S.A.

\begin{abstract}
The Extreme Ultraviolet Explorer data archive has been used to study flare energy distributions and infer the role of flares in coronal heating. We have constructed flare number distributions as a function of observed EUV and X-ray emitted thermal energy. We find cumulative flare distributions of the form $N(>E) \propto E^{-\alpha+1}$ with $\alpha \approx 1.5-2.6$. We present results in the context of spectral type classification.
\end{abstract}

To explain coronal temperatures (1-30 MK), it has been proposed that flares play a crucial role (e.g., Parker 1988). Flares have been found to be distributed in energy according to a power law, i.e., $\mathrm{d} N / \mathrm{d} E \propto E^{-\alpha}$, with $\alpha$ around 2 . Hudson (1991) argued that, if $\alpha>2$ for solar microflares, an extrapolation to lower energies may provide the energy necessary to heat the whole corona. We have initiated a project in which we analyze data from the EUVE archive to study coronal heating on active late-type stars. Audard, Güdel, \& Guinan (1999) found indices $\alpha$ for two young, active solar analogs that suggest that their coronae could be heated by flares. Here, we present an extension to 10 additional data sets.

First method: We reduced stellar data from the EUVE archive. A flare identification method based on the count distribution of "quiescent" radiation and the probability of Poisson occurrence was then applied (see Audard et al. 1999; 2000). Using fits to the light curves, we derived the flare durations and emitted energies. Finally, flare number distributions in emitted thermal energy (0.01$10 \mathrm{keV}$ ) were constructed and fitted (Figure 1, Table 1; Audard et al. 2000).

Second method: Another method was applied to the data of the active binary FK Aqr (dM2e+dM2e). The pattern of observed photon arrival times is compared with model photon events (Drake et al., in preparation). The derived index $\alpha \approx 2.4$ suggests that FK Aqr's flares follow a steep distribution.

\footnotetext{
${ }^{1}$ Also at: Institute of Astronomy, ETH Zentrum, 8092 Zürich, Switzerland
} 

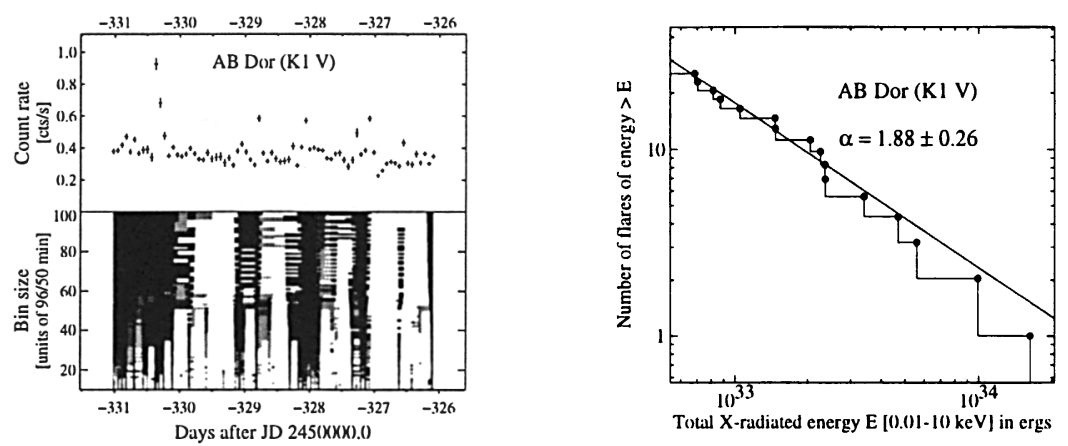

Figure 1. An example of our procedure results (for AB Dor).

Furthermore, a possible trend was discovered in which M-type stars have lower power-law indices $\alpha$ than F- and G-type stars, suggesting that the latter could have their coronae heated by flares. Further studies will be presented in Audard et al. (2000).

Table 1. Results of the different fitting methods.

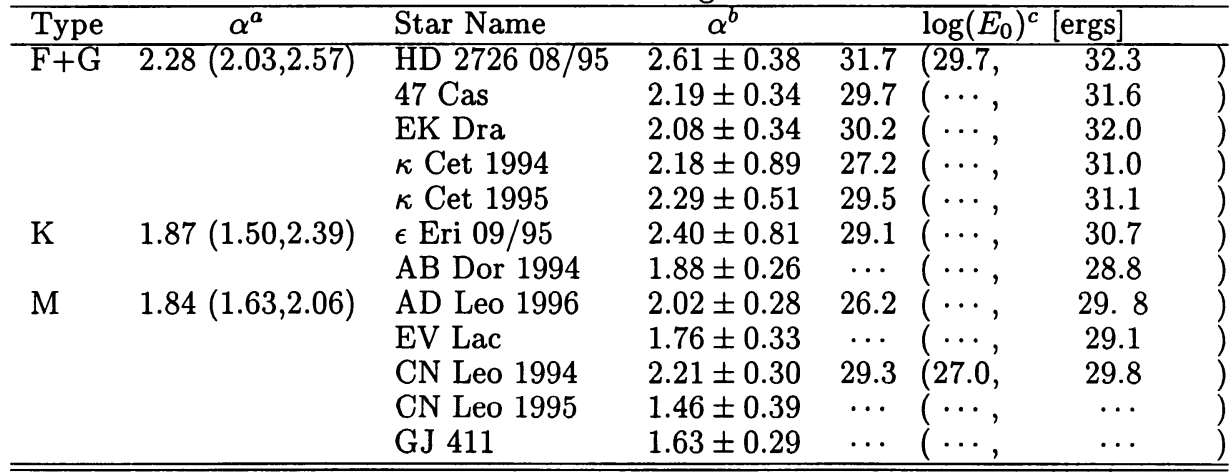

${ }^{a}$ For differential distributions with $68 \%$ confidence ranges for 1 parameter.

${ }^{b}$ For cumulative distributions, from an adapted version of Crawford, Jauncey, \& Murdoch (1970).

$c$ Minimum energy required to explain the total observed radiative energy loss.

Acknowledgments. We are thankful to K. Arnaud for his help. M. A. acknowledges support from the Swiss National Science Foundation, grant 2149343.96, and from the Swiss Academy of Sciences. All data were obtained from the Multimission Archive at STScI, which is operated by AURA, Inc.

\section{References}

Audard, M., Güdel, M., Drake, J. J., \& Kashyap, V. L. 2000, ApJ, submitted Audard, M., Güdel, M., \& Guinan, E. F. 1999, ApJ, 513, L53

Crawford, D. F., Jauncey, D. L., \& Murdoch, H. S., 1970, ApJ, 162, 405

Drake, J. J., Kashyap, V., Audard, M., \& Güdel, M., in preparation

Hudson, H. S. 1991, Solar Phys., 133, 357

Parker, E. N. 1988, ApJ, 330, 474 